\title{
Evaluation of Employees' Competence in Transnational Corporations Based on Complex Network Theory
}

\author{
Zi-Jun HUANG ${ }^{1, a,{ }^{*},}$ Tian DONG ${ }^{1, b}$ \\ ${ }^{1}$ Beijing University of Posts and Telecommunications, School of Science, Beijing, China \\ aalfredhuangzj@gmail.com, balexia18612187331@gmail.com \\ *Corresponding author
}

Keywords: System Management Theory, Complex network theory, Centrality, Influence attenuation function, Standard feature vector with weight, Sensitivity analysis.

\begin{abstract}
This paper aims at constructing a complex network to evaluate employees' competence in transnational corporations. By analyzing network's structure, weight vectors are constructed which evaluate the degree of importance among factors such as relative degree centrality, relative betweenness centrality and relative closeness centrality. Therefore, a score function model is proposed. This model is then modified by introducing standardized eigenvectors and influence reduction function. Sensibility of this model is also defined to assess employees' competence in transnational companies. A reference method is given to measure the ability of employees and to give some advice on training their staff for some important positions.
\end{abstract}

\section{Introduction}

The ability of employees has a great impact on enterprise competence, therefore, a proper model has to be established to evaluate employees' performance. Moreover, in China, those research methods mainly based on questionnaire are relatively simple and lack diversity. Referring to the popularity of communicating by e-mails which has built a network among employees, this study will provide a measurable gist for management in transnational corporations by objective data analysis.

\section{The establishment of the evaluation system}

\section{The establishment of the graph of relationship}

Building up a network lies first in the establishment of evaluation system. This paper evaluates relationship among employees by e-mails they send or receive. First of all, two relationships need to be given definitions about e-mails and communication:

a)A sending process will only be defined as a complete one when an e-mail is read by recipients. Unread e-mails will not be discussed.

b) Communication is bidirectional. The information is defined as invalid in e-mail communication if a sends $b$ an e-mail but b does not reply.

Then a graph $G_{e}(\mathrm{~V}, \mathrm{R})$ without direction and edge weight can be defined. $\mathrm{V}$ is the set of vertexes and $\mathrm{R}$ the relationship among all vertexes. Thus a complicated network is formed.

\section{The factors of evaluation}

The ability of employees will be evaluated by indexes below:

a) Relative degree centrality: the biggest probable ratio of degree of absolute degree centrality and points in this graph. Degree of absolute degree centrality is the actual degree of this point.

b) Relative betweenness centrality: a standardized absolute betweenness centrality. Betweenness centrality of one point is the ability that this point on this geodesic.

c) Relative closeness centrality: a standardized absolute closeness centrality. The closeness centrality is the sum of distances between this point and other points on this graph.

d) Knowledge: an evaluation of the knowledge and learning ability of an employee 
e) Quality: an evaluation of the attitude and methods of an employee when he deals with affairs

As for the first three factors, Freeman's view is:” This depends on the background of research. If focus is the communicational activity, degree centrality can be used as a criterion. If focus is the control of communication, betweenness centrality can be adopted. If dependence or effectiveness of information delivery is analyzed, closeness centrality can be used.” [1]

Three different centralities have their own focuses, and their weight will be distributed by AHP in this paper. The latter two factors could be analyzed further by AHP, for example, quality can be evaluated by the sense of responsibility and honor, the ability of cooperation and the attitude towards learning, while knowledge may be decided as the application of knowledge, professional knowledge and common sense and so on.[2]

Evaluation of the employees' competence by radar

The employees' competence can be evaluated roughly by radar map according to the five factors above.

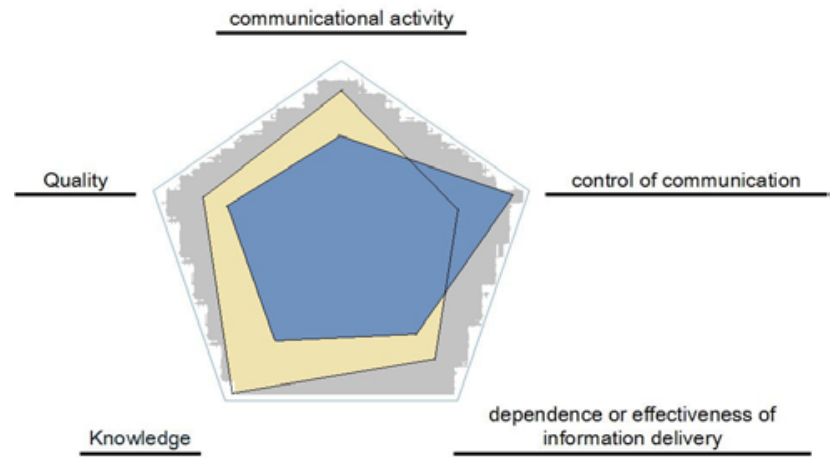

Fig. 1 the comparison between "yellow" employees and "blue” employees

\section{Evaluation of the employees' competence by constructing score functions}

The above five factors constitute a vector, marked as $\left(\begin{array}{lllll}C_{r d} & C_{r b} & C_{r p} & K & Q\end{array}\right)$, and weight of these factors are $\left(\begin{array}{lllll}\partial_{1} & \partial_{2} & \partial_{3} & \partial_{4} & \partial_{5}\end{array}\right)^{\prime}$. Therefore, the score function expression is

$$
s=\left(\begin{array}{lllll}
C_{r d} & C_{r b} & C_{r p} & K & Q
\end{array}\right)\left(\begin{array}{lllll}
\partial_{1} & \partial_{2} & \partial_{3} & \partial_{4} & \partial_{5}
\end{array}\right)^{\prime}
$$

The latter two factors are difficult to get, so they are omitted in this model. ( If they can be got in later research, they can constitute the score functions by the same model.) So the score function expression is:

$$
S=\left(\begin{array}{lll}
C_{r d} & C_{r b} & C_{r p}
\end{array}\right)\left(\begin{array}{lll}
\partial_{1} & \partial_{2} & \partial_{3}
\end{array}\right)^{\prime}
$$

marked as “*”.

\section{The improvement of the evaluation system}

The model above does not take some factors into consideration, such as the number of the e-mails that one sends and receives. So the model will be improved from the following two aspects.

\section{The direct interplay of people}

There are many examples in our daily life. If one is regarded as entitled, then he or she will become more entitled. A person's influence and other relevant persons' influence constitute a function.

In the analysis of social network, the core node can be found by the calculation of the eigenvectors, and the eigenvectors have little to do with the local structure. Moreover, in the research of social 
network, the first dimension may integrate the properties of all the dimensions and the second and other dimensions have more specific and partial properties according to the features of factor analysis.

Given $\mathrm{A}$ is an adjacent matrix, the element is $a_{i j}$ the contribution measuring the amount of influence that giver $i$ has given to $j$. Given $\mathrm{x}$ as the vector of degree centrality then the statement above can be expressed as:

$x_{i}=a_{1 i} x_{1}+a_{2 i} x_{2}+\cdots+a_{n i} x_{n}$

That is, the centrality of one person is a function of the centralities of other persons who have chosen him. The matrix is:

$A^{t} x=x, A^{t}$ is the transposed matrix of $A$

In the formula (4), $\mathrm{X}$ is an eigenvector corresponding with the eigenvalue one. Usually, the formula (4) has no untrivial solution, unless A has an eigenvalue one. These equation sets will have solutions if every line in the matrix is standardized, so that the sum of each line is one. Then the formula (4) has untrivial solutions as A has an eigenvalue one. Another method is to generalize formula (4) to a eigenvector function in a general sense, and replace formula (3) and formula (4) with formula (5) and formula (6) respectively:

$$
\lambda x_{i}=a_{1 i} x_{1}+a_{2 i} x_{2}+\cdots+a_{n i} x_{n}
$$

$A^{t} x=\lambda x$

If $A$ is a matrix $n \times n$, the formula (6) will have $n$ solutions corresponding $n \lambda$. The general type of solution can be expressed by matrix as: $A \cdot \mathrm{X}=\lambda \cdot \mathrm{X}$. X is a matrix: $n \times n$ Each line of matrix $\mathrm{A}$ is an eigenvector of $A$, and $\lambda$ is a diagonal matrix composed by every eigenvalue. [3]

Suppose that the more e-mails one sends and receives the more influential one will be. Certainly, the number of e-mails one employee sends and receives is not completely equal to his or her influence, and their weight can be affirmed by experts' judgment. Given that there are nine portions of influence out of ten portions come from the number of received e-mails, and one portion comes from the sent e-mails, an expression that defines the influence of an employee a will be

$$
P I_{a}=0.9 \cdot N_{\text {in }}+0.1 \cdot N_{\text {out }}
$$

Then the expression that defines average influence will be

$$
\overline{P I}_{a \rightarrow i}=\frac{0.9 \cdot N_{\text {in }}+0.1 \cdot N_{\text {out }}}{L O}
$$

in which $\overline{P I}_{a \rightarrow i}$ is the average influence that a gives to $i$, and $L O$ is the degree of a in network graph. Define a matrix $A$ which has a scale of $\mathrm{N}, A=\left(a_{i j}\right)_{N \cdot N}, a_{i j}$ is the influence that employee $i$ have on employee $j$, (that is $a_{i j}=\overline{P I}_{i \rightarrow j}$ ), and $a_{i i}=0(\mathrm{i}=1,2, \cdots)$, which means the influence that one have on himself is not taken into consideration. According to the weight of eigenvalue, the score formula that has been defined again is: 
$S_{m}=\left(\begin{array}{lll}C_{r d} & C_{r b} & C_{r p}\end{array}\right)\left(\begin{array}{lll}\partial_{1} & \partial_{2} & \partial_{3}\end{array}\right)^{\prime} \cdot \mathrm{w}_{i j}$

in which $\mathrm{w}_{i j}$ is the standardized eigenvector. Therefore, the discrimination among different numbers of e-mails is realized by weighing scores.

In order to prevent situation when the final score may be zero, a minor number is put in front of $\mathrm{w}_{i j}$. (In practice, different number can be put according to different models). Then the score function expression can be

$S_{m}=\left(\begin{array}{lll}C_{r d} & C_{r b} & C_{r p}\end{array}\right)\left(\begin{array}{lll}\partial_{1} & \partial_{2} & \partial_{3}\end{array}\right)^{\prime} \cdot\left(\mathrm{w}_{i j}+0.05\right)$

\section{The indirect interplay of people}

Based on 2.1, this section will perfect the value of $\overline{P I}_{a \rightarrow i}$ The following assumptions are considered as reasonable: if a has not communicated with c, but a and c both has communicated with $\mathrm{b}$, then $\mathrm{a}$ is influencing $\mathrm{c}$ (or $\mathrm{c}$ is influencing a). This influence is called individual's average indirect influence, marked as $\overline{P I}_{i \rightarrow j}(\mathrm{n})$, in which $\mathrm{n}$ is the degree of indirection which is called the degree of alienation. In the above example, the value of $n$ is one. When $n$ takes a value of $0, \overline{P I}_{i \rightarrow j}(0)$ is equal to the $\overline{P I}_{i \rightarrow j}$ in 2.1. This indirect influence can be accumulated. That is, when calculating $\overline{P I}_{i \rightarrow j}$ (2), the influence of $i$ becomes bigger due to the influence of $\overline{P I}_{h \rightarrow i}, h$ are the points whose degree of alienation to $i$ is one in the network.

The influence of a is spreading around $a$, and the influence is reducing in proportion during spreading, which resembles much the proportional decay of radioactive elements in physics so an existing physical model is adopted in describing the degree of influence reduction. [4] Given the degree of alienation as $\mathrm{n}$, then the average influence that a has on $j$ is

$$
\overline{P I}_{i \rightarrow j}(\mathrm{n})=\frac{\overline{P I}_{i \rightarrow j} \times f(n)}{L O}
$$

in which $\mathrm{f}(\mathrm{n})=e^{-n}$ Meanwhile, the remediated expression of $i$ 's influence on $j$ is

$$
\overline{F P I}_{i \rightarrow j}=\sum_{k=0}^{\infty} \overline{P I}_{i \rightarrow j}(\mathrm{k})
$$

In practical operation, when $n \geq 2, \mathrm{f}(\mathrm{n}) \leq 0.05$. The indirect influence can be neglected naturally, So the final expression is

$$
\overline{F P I}_{i \rightarrow j}=\overline{P I}_{i \rightarrow j}(0)+\overline{P I}_{i \rightarrow j}(1)+\overline{P I}_{i \rightarrow j}(2)
$$

When there is no opportunity for misunderstanding the meaning, $\overline{F P I}_{i \rightarrow j}$ is called the influence that $i$ has on $j$. Replace $\overline{P I}_{i \rightarrow j}$ with $\overline{F P I}_{i \rightarrow j}$, the score function expression is:

$$
S=\left(\begin{array}{lll}
C_{r d} & C_{r b} & C_{r p}
\end{array}\right)\left(\begin{array}{lll}
\partial_{1} & \partial_{2} & \partial_{3}
\end{array}\right)^{\prime} \cdot\left(\mathrm{w}_{i j}{ }^{\prime}+0.05\right)
$$

in which $\mathrm{w}_{i j}{ }^{\prime}$ is the standardized eigenvector, marked as “***”. 


\section{Definition of sensitivity}

In order to evaluate our model, definition of sensitivity in this model: the amount of variation on ranking by increasing or decreasing a node. To be specific, denote the ranking (position) of the ith person in the initial ranking system as $r_{i}(1 \leq i \leq N)$, and thus form a column vector, namely $r$. Every node reduced will influence the its ranking. The new ranking will be calculated according to the assessment system in the model, denoting as $r^{\mathrm{m}}(\mathrm{i})$, where $i$ is the position of node canceled. In the rearranged column vector, the node discarded is placed on the final position. (This is also a realistic setting, because after a node is canceled its degree becomes zero. In this assessment system, it definitely scores zero. At this point, the sensitivity of the model is denoted as

$$
S e=\frac{\left\|r^{m}(\mathrm{i})-r\right\|_{2}^{2}}{N}
$$

If a node is added, the new column vector is denoted as $r^{a}(j)$. Similarly, the node added is placed on the final position. In this situation, sensitivity of the model is denoted as

$$
S e=\frac{\left\|r^{a}(\mathrm{j})-r^{\prime}\right\|_{2}^{2}}{N+1}
$$

In conclusion,

$$
S e=\left\{\begin{array}{l}
\frac{\left\|r^{m}(\mathrm{i})-r\right\|_{2}^{2}}{N}, \text { delete } \\
\frac{\left\|r^{a}(\mathrm{j})-r^{\prime}\right\|_{2}^{2}}{N+1}, \text { add }
\end{array}\right.
$$

in which

$$
r=\left(\begin{array}{c}
r_{1} \\
r_{2} \\
r_{3} \\
\vdots \\
r_{N-2} \\
r_{N-1} \\
r_{N}
\end{array}\right)_{N \times 1} \quad r^{\mathrm{m}}(\mathrm{i})=\left(\begin{array}{c}
r_{1}^{m} \\
\vdots \\
r_{i-1}^{m} \\
N \\
r_{i+1}^{m} \\
\vdots \\
r_{N}
\end{array}\right)_{N \times 1} \quad r^{\prime}=\left(\begin{array}{c}
r_{1} \\
r_{2} \\
r_{3} \\
\vdots \\
r_{N-2} \\
r_{N-1} \\
r_{N} \\
N+1
\end{array}\right)_{(\mathbb{N}+1) \times 1} \quad r^{a}(\mathrm{j})=\left(\begin{array}{c}
r_{1}^{a} \\
r_{2}^{a} \\
r_{3}^{a} \\
\vdots \\
r_{N-2}^{a} \\
r_{N-1}^{a} \\
r_{N}^{a} \\
r_{j}^{a}
\end{array}\right)_{(\mathrm{N}+1) \times 1}
$$

\section{The example of evaluation}

\section{The construction and result of the model}

Take an transnational enterprise as an example where a e-mail receiving and sending network of 466 employees can be obtained by consultation and investigation. Adopting the methods discussed in this paper, the adjacent matrix and the network of relationships among employees can be got with help of Java to extract data and Graphi to make a graph: 


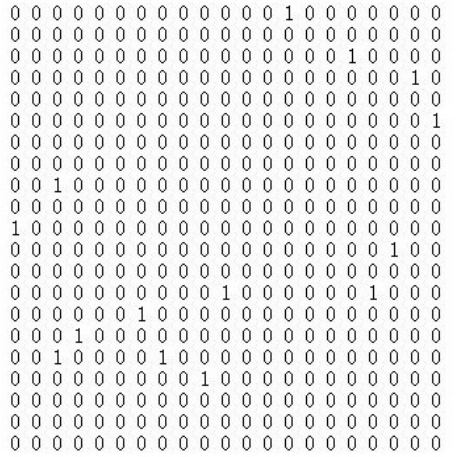

Fig. 3 network of the relationships of employees

Use Ucinet to calculate three centralities of each node and the standardized eigenvectors in the final model, and use AHP to distribute the weight of these factors $\left(\begin{array}{lll}\partial_{1} & \partial_{2} & \partial_{3}\end{array}\right)^{\prime}$, the ranking of employees which depends on the score can be obtained according to the above three expressions. [5]

Tab. 1 the weight got by AHP

\begin{tabular}{cc}
\hline Factors & Weight \\
\hline Relative degree centrality & 0.38 \\
Relative betweenness centrality & 0.33 \\
Relative closeness centrality & 0.29 \\
\hline
\end{tabular}

Tab. 2 the ranking of scores that employees get according to these three expressions

\begin{tabular}{lllllllllll}
\hline S/Ranking & 1 & 2 & 3 & 4 & 5 & 6 & 7 & 8 & 9 & 10 \\
$*$ & 9 & 170 & 150 & 400 & 135 & 40 & 437 & 409 & 346 & 311 \\
$* *$ & 118 & 368 & 400 & 351 & 164 & 364 & 393 & 54 & 40 & 422 \\
$* * *$ & 118 & 400 & 368 & 364 & 164 & 351 & 40 & 437 & 393 & 135 \\
\hline
\end{tabular}

Remark: The number in this table is the ID of employees. “*”, ”**”, “***” represent respectively the three score expressions discussed in this paper.

Combining with feedback given by the company, the ranking which is got according to“***“ is more consistent with actual situation. That is the companies can manage their employees in a measurable way according to the method provided by this paper.

\section{The sensitivity of the model}

Analyze the corporation according to this model. The figure below shows that model has good robustness in most cases. However, if key nodes (as shown point D in figure) are under malicious attacks, this model will be vulnerable owing to the structure and characteristics of net. For companies, these important nodes ( or important posts) are necessary for a stable development so that companies should attach more importance on these posts. 


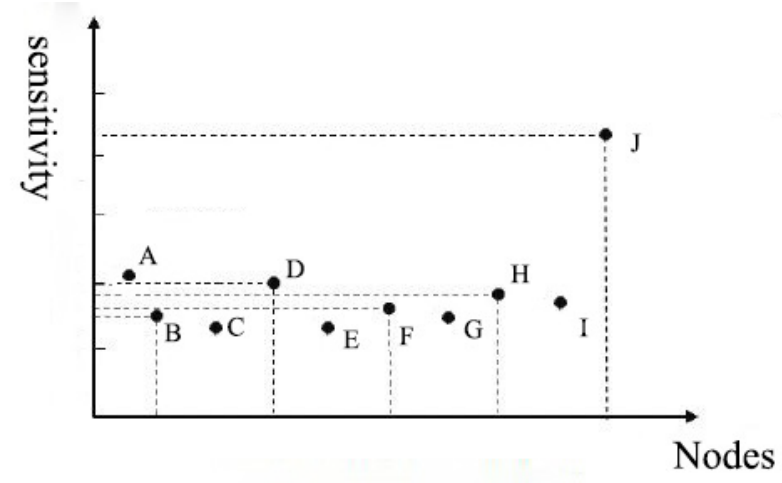

Fig. 4 the sensitivity of model when some nodes are randomly deleted or added

\section{Summary}

The paper gives a reference method which measures the ability of employees and gives some advice on training their staff for some important positions.

\section{Acknowledgement}

Many people have made invaluable contributions, both directly and indirectly to my research. I would like to express my warmest gratitude to Prof. Guo yucui, my supervisor, for her instructive suggestions and valuable comments on the writing of this thesis. Without her invaluable help and generous encouragement, the present paper would not have been accomplished. Also, I want to say Thank you to Research Innovation Fund for College Students of Beijing University of Posts and Telecommunications (X-201310013066) and National University Student Innovation Program, which support this research financially.

\section{References}

[1]Freeman, L.C., 1979, Centrality in Social Networks: Conceptual Clarification, Social Networks. $1,215-239$

[2]Shaomei Yang, Analytic Hierarchy Process (AHP) in the Application of the Employee Performance Evaluation, Journal of North China Electric Power University Vol.33, No.4 Jul. 2006 http://wenku.baidu.com/link?url=HMplrjhzz4Ikf5vUD1wNDnFlYwOFYQqPPPf60RsU10vjj-Un95 UXpqLgml1qkyKk07zmNiziolI5MXP_bczBohTdp4706vaGc3dzJ2_fAvW

[3]Jun Liu, Social Network Model Research, Sociological Research, 2004(1)

http://wenku.baidu.com/view/9eec501ba76e58fafab0031c.html

[4] Information on http://en.wikipedia.org/wiki/Radioactive_decay

[5] Borgatti S.P., Everett M.G., Freeman L.C. (2002): UCINET 6 for Windows. Harvard: Analytic Technologies. http://www.analytictech.com/ 